\title{
Impacto de la contingencia sanitaria por COVID-19 en las estrategias de reperfusión del síndrome coronario agudo
}

\author{
Impact of COVID-19 on reperfusion strategies for acute coronary syndromes \\ Gabriela Torres-Escobar, Héctor González-Pacheco, Jose Luis Briseño-De la Cruz, \\ Alexandra Arias-Mendoza y Diego Araiza-Garaygordobil* \\ Unidad Coronaria, Instituto Nacional de Cardiología Ignacio Chávez, Secretaría de Salud, Ciudad México, México
}

\begin{abstract}
Resumen
Las terapias de reperfusión, tales como intervención coronaria y fibrinólisis, son las principales medidas de atención en pacientes con síndromes coronarios agudos. La angioplastia primaria se considera el estándar de oro, sin embargo, en pacientes con infección por coronavirus 2 del síndrome respiratorio agudo grave (SARS-CoV-2), la estrategia de reperfusión más recomendada es la terapia fibrinolítica, debido al menor tiempo requerido para realizarla y menor exposición al agente infeccioso. Esta pandemia representa una problemática de contagio en el personal de salud, ya que los casos van en aumento a nivel mundial, por lo cual es importante conocer las medidas que se deben seguir a fin de evitar la enfermedad por coronavirus 2019 (COVID-19).
\end{abstract}

Palabras clave: COVID-19. Pandemia. Fibrinólisis. Angioplastia. STEMI. NSTEMI.

\begin{abstract}
Reperfusion therapy is a measure of care in patients with ST-elevation myocardial infarction (STEMI), which should be performed once we have the diagnosis. Percutaneous coronary intervention is considered the gold standard, however in patients with SARS-CoV-2 infection, the reperfusion strategy is more focused on fibrinolytic therapy due to the shorter time required to perform and less exposure. This pandemic represents a contact problem in health personnel, since cases are increasing worldwide, so it is important to know the measures that must be followed to avoid coronavirus disease (COVID-19).
\end{abstract}

Key words: COVID-19. Pandemic. Fibrinolysis. Angioplasty. STEMI. NSTEMI.

\section{Introducción}

La intervención coronaria percutánea (ICP) y la fibrinólisis representan estrategias terapéuticas enfocadas en la reperfusión coronaria en pacientes con síndromes coronarios agudos (SCA). La terapia fibrinolítica fue el primer tratamiento de reperfusión efectivo que se implementó sistemáticamente, pero tiempo después la ICP demostró ser superior, convirtiéndose en el estándar de atención en todo el mundo ${ }^{1}$, al reducir

\section{Correspondencia:}

*Diego Araiza-Garaygordobil

Juan Badiano, 1

Col. Belisario Domínguez, Sección XVI, Tlalpan

Fecha de recepción: 22-04-2020

Fecha de aceptación: 30-04-2020

Péxico, México

E-mail: dargaray@gmail.com

DOI: 10.24875/ACM.M20000069 CC BY-NC-ND (http://creativecommons.org/licenses/by-nc-nd/4.0/). 
la mortalidad, los reinfartos o los eventos vasculares cerebrales $^{2}$. La actual contingencia sanitaria mundial producida por el nuevo coronavirus 2 del síndrome respiratorio agudo severo (SARS-CoV-2), causante de la enfermedad por coronavirus 2019 (COVID-19), tiene altas tasas de mortalidad ( $15 \%$ en pacientes ancianos e inmunocomprometidos) y contagio, por lo cual la atención de pacientes con SCA debe dirigirse a disminuir la exposición y propagación.

Uno de los principales desafíos en esta pandemia es el diagnóstico de SCA, ya que ambas condiciones pueden coexistir y los síntomas superponerse, sobre todo si consideramos que la mayoría de las pruebas de detección COVID-19 pueden ser negativas (30\% falsas negativas) y $<80 \%$ de las infecciones son asintomáticas. De acuerdo con la experiencia generada por la epidemia de COVID-19 en China, se ha recomendado la realización de terapia fibrinolítica en lugar de ICP para infarto con elevación del segmento ST (STEMI), a fin de permitir el desarrollo de un cuadro clínico completo del proceso infeccioso ${ }^{2}$.

\section{Terapia fibrinolítica}

El estudio STREAM es un ensayo clínico contemporáneo que reclutó pacientes con STEMI $\leq 3$ horas de inicio de los síntomas que no pudieron acceder a ICP primaria $\leq 1$ hora del primer contacto médico. Los pacientes fueron asignados al azar a terapia trombolítica, con ICP después de 6-24 horas o ICP primaria con diferencia media entre la administración de fibrinolíticos e ICP $\geq 78$ minutos. Los resultados de la terapia fibrinolítica vs. ICP primaria fueron similares para el compuesto de mortalidad, choque, insuficiencia cardiaca o reinfarto. Cabe resaltar que la necesidad de angiografía emergente en el brazo fibrinolítico fue del $36 \%$, la mortalidad fue menor del $5 \%$ en ambos grupos y la hemorragia intracraneal fue mayor con terapia fibrinolítica ( 1.0 vs. $0.5 \%$; $p=0.02$ ), lo cual indicó que cuando la demora en ICP primaria es inevitable, un enfoque farmacoinvasivo puede ser adecuado en la era de los inhibidores P2Y12².

\section{Retraso de la ICP primaria}

Este tiempo puede ser dependiente de varios elementos, principalmente del sistema de atención y de los operadores individuales, incluso entre pacientes negativos para SARS-CoV-2, debido al tiempo necesario para recolectar un historial clínico y síntomas antes de su ingreso a una sala de hemodinamia, por lo que el personal requiere tiempo para colocarse equipo de protección personal y realizar sus funciones habituales. Por estos factores, la administración inmediata de fibrinolíticos puede disminuir los retrasos basados en sistemas, sobre todo teniendo en cuenta que un tiempo puerta-aguja de 30 minutos es más factible que un tiempo puerta-balón de 90 minutos $^{2}$. En estos casos en específico se debe mencionar que la reperfusión temprana podría ser más importante que el modo de reperfusión.

\section{Problemática de la COVID-19 en otros países enfocada a personal hospitalario}

Al ser altamente contagioso, con propagación dependiente de la proximidad y viabilidad en aerosoles durante horas o superficies durante días, la infección de los trabajadores de la salud es preocupante. En Italia y España el $8-12 \%$ de los infectados son trabajadores de la salud, por lo cual se debe enfatizar que el uso de equipos de protección individual minimiza el riesgo pero no lo elimina y probablemente un factor importante sea el tiempo de exposición².

\section{Estrategias en SCA y COVID-19}

1) Todos los pacientes STEMI deben someterse inicialmente a pruebas clínicas COVID-19.

2) En pacientes con STEMI y confirmación de COVID-19, el aislamiento debería ser inmediatamente realizado, además de evaluarse las contraindicaciones para trombólisis ${ }^{3}$.

3) En los pacientes con riesgo elevado y contraindicación para la terapia trombolítica debe valorarse la relación entre riesgo y beneficio de la ICP.

4) En pacientes con STEMI y sospecha de COVID-19 (definida como fiebre, síntomas respiratorios y antecedentes de contacto con otros casos de COVID-19), el examen sanguíneo ordinario, la reacción en cadena de la polimerasa (PCR), el examen de esputo para la detección de coronavirus y la tomografía axial computarizada de tórax deberían ser realizadas para la confirmación de COVID-19, no obstante que la decisión para la terapia de reperfusión debe iniciarse dentro de los primeros 30 minutos de haberse realizado el diagnóstico de STEMI.

5)Si el diagnóstico de COVID-19 fue realizado posterior a la terapia fibrinolítica, el paciente debe derivarse a una institución médica encargada de enfermedades infecciosas. 


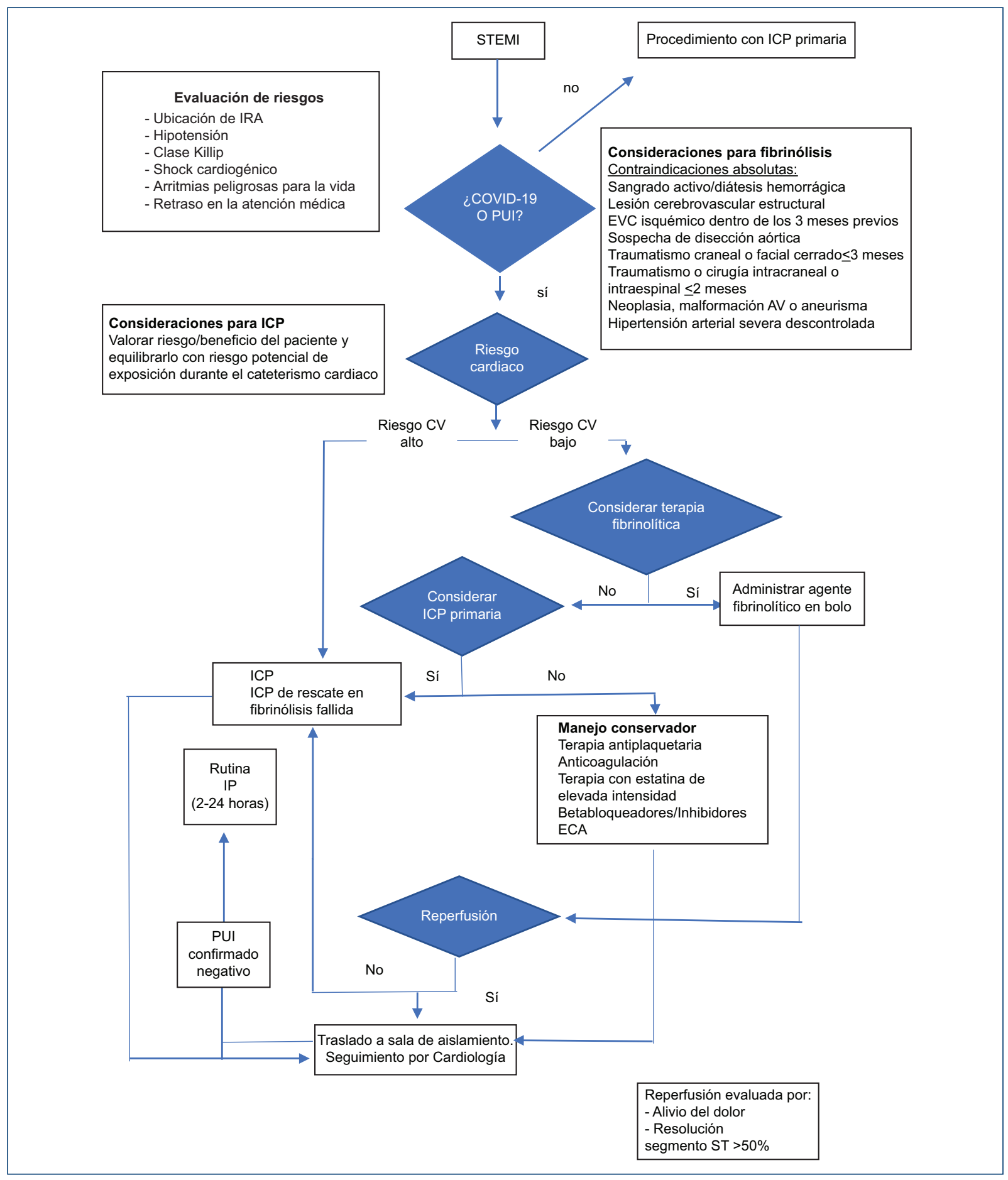

Figura 1. Algoritmo manejo de STEMI.

6)En pacientes con STEMI y bajo riesgo de COVID-19 (definido como la ausencia de fiebre, síntomas respiratorios y antecedente de exposición a otros casos de COVID-19) el examen sanguíneo ordinario, electrocardiograma, tomografía de tórax, los biomarcadores de lesión miocárdica y el ecocardiograma en la cabecera del paciente deberían ser considerados de forma inmediata ${ }^{3}$.

7)En caso de complicaciones, los procedimientos de cabecera son preferibles (bomba de balón intraaórtico, pericardiocentesis, oxigenación por membrana extracorpórea, marcapasos venosos temporales) ${ }^{3}$. 


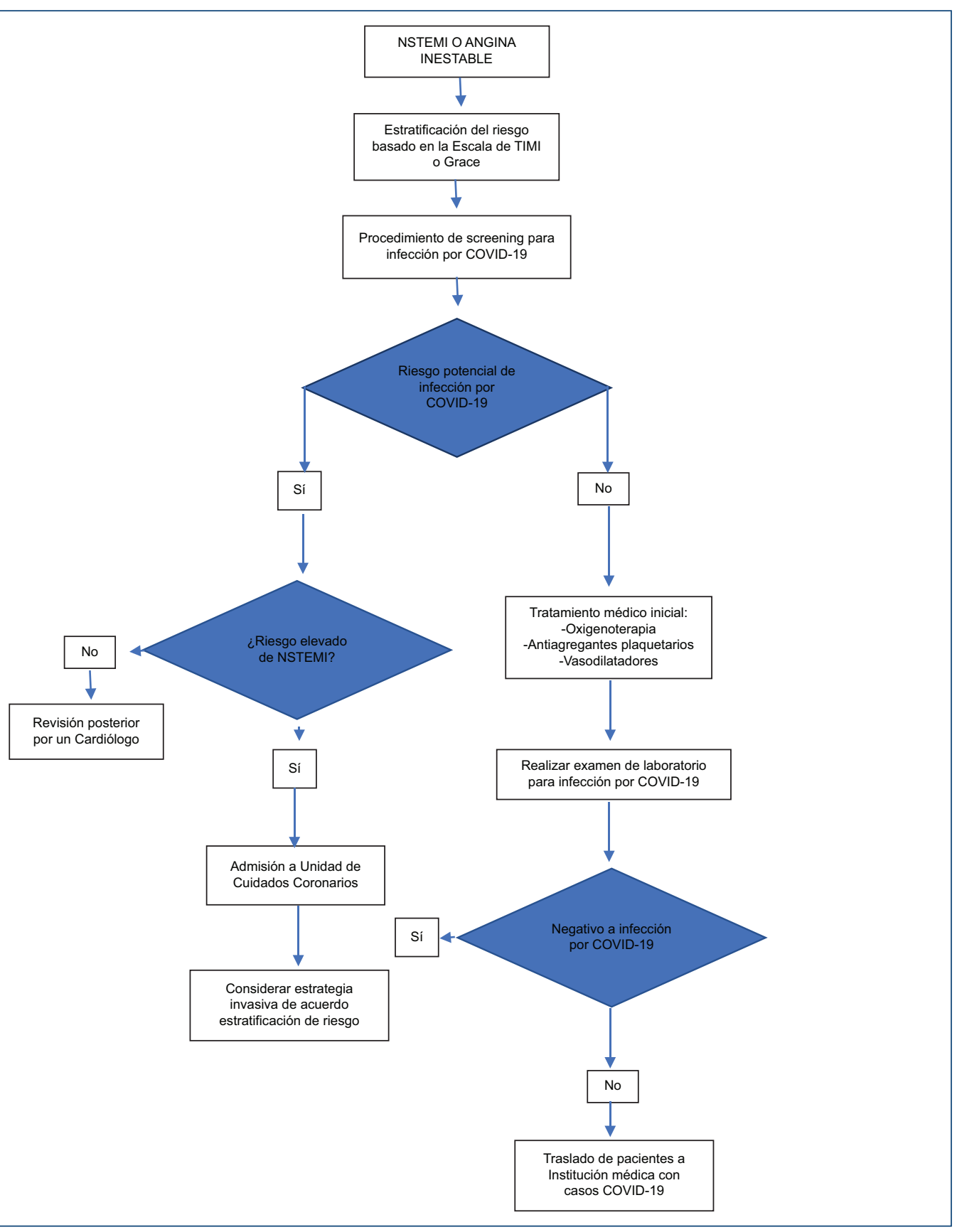

Figura 2. Algoritmo de manejo NSTEMI o angina inestable.

8) Para el tratamiento en la sala de hemodinamia se debe emplear la máxima protección para evitar la exposición del personal (equipos de protección individual).

9) En caso de ICP, esta solo debe realizarse en el vaso culpable a menos que una lesión no culpable se considere inestable o existan múltiples lesiones culpables.

10) Evitar la intubación en la sala de hemodinamia, así como el uso de la cánula nasal de alto flujo, la ventilación no invasiva y el uso de bolsa válvula mascarilla a fin de evitar la aerosolización y diseminación del virus.

Estrategias en infarto agudo de miocardio sin elevación del segmento ST y COVID-19

La terapia de reperfusión en pacientes con infarto agudo de miocardio sin elevación del segmento ST 
Tabla 1. Uso del equipo de protección personal de acuerdo con el nivel de atención

\begin{tabular}{|c|c|c|c|c|c|c|}
\hline \multicolumn{7}{|c|}{ Uso de equipo de protección personal de acuerdo con el nivel de atención } \\
\hline Nivel de caso & $\begin{array}{c}\text { Higiene } \\
\text { de manos }\end{array}$ & Bata & $\begin{array}{l}\text { Máscara } \\
\text { médica }\end{array}$ & $\begin{array}{l}\text { Respirador } \\
\text { (N95 o FFP2) }\end{array}$ & $\begin{array}{l}\text { Gafas (protección ocular) } \\
\text { o protector facial }\end{array}$ & Guantes \\
\hline Triaje & $x$ & & $x$ & & & \\
\hline $\begin{array}{l}\text { Recolección de muestras para } \\
\text { diagnóstico de laboratorio }\end{array}$ & $x$ & $x$ & & $x$ & $x$ & $x$ \\
\hline $\begin{array}{l}\text { Caso sospechoso o confirmado de } \\
\text { COVID-19 que requiere admisión a un } \\
\text { centro de salud y NO requiere } \\
\text { procedimiento generador de aerosoles }\end{array}$ & $\mathrm{x}$ & $\mathrm{x}$ & $x$ & & $\mathrm{x}$ & $\mathrm{x}$ \\
\hline $\begin{array}{l}\text { Caso sospechoso o confirmado de } \\
\text { COVID-19 que requiere admisión a un } \\
\text { centro de salud y Sí requiere } \\
\text { procedimiento generador de aerosoles }\end{array}$ & $\mathrm{x}$ & $x$ & & $\mathrm{x}$ & $\mathrm{x}$ & $\mathrm{x}$ \\
\hline
\end{tabular}

(NSTEMI) no tiene beneficio clínico. Las estrategias de tratamiento deben determinarse con base en la estratificación de riesgo de la enfermedad arterial coronaria. La escala GRACE debe utilizarse mientras se esperan los resultados de la PCR, y una vez confirmados, derivarse a una institución médica especializada en enfermedades infecciosas, inmediatamente ${ }^{4}$. En tanto que si la infección por SARS-CoV-2 puede ser excluida, está indicado el cambio en la estrategia de tratamiento de acuerdo con la estratificación del riesgo.

\section{Conclusiones}

La infección por SARS-CoV-2 y el SCA pueden sobreponerse en algunos pacientes. Los estudios publicados de dicha asociación actualmente son escasos, ya que la COVID-19 se trata de una enfermedad emergente. No obstante, debemos estar atentos a la manifestación de síntomas de sospecha en ambos padecimientos, y de ser posible, realizar estudios diagnósticos que nos permitan orientar el manejo en estos pacientes de manera oportuna. Es importante además, que ante la sospecha de infección por SARS-CoV-2, se tomen las medidas necesarias en el personal de salud a fin de evitar contagios dentro de la unidad hospitalaria. Por último, hay que resaltar que en el STEMI se tiene que optar por el método de reperfusión que se pueda administrar con mayor prontitud, por lo que la balanza en estos momentos se inclinaría hacia el uso de trombolíticos.

\section{Responsabilidades éticas}

Protección de personas y animales. Los autores declaran que para esta investigación no se han realizado experimentos en seres humanos $\mathrm{ni}$ en animales.

Confidencialidad de los datos. Los autores declaran que han seguido los protocolos de su centro de trabajo sobre la publicación de datos de pacientes.

Derecho a la privacidad y consentimiento informado. Los autores han obtenido el consentimiento informado de los pacientes y/o sujetos referidos en el artículo. Este documento obra en poder del autor de correspondencia.

\section{Bibliografía}

1. Daniels M, Cohen M, Bavry A, Kumbhani D. Reperfusion of STEMI in the COVID-19 Era - Business as usual? Circulation. 2020 Apr 13. doi: 10.1161/CIRCULATIONAHA.120.047122. [Epub ahead of print]

2. Ibáñez B, James E, Agewall, Antunes M, Bucciarelli $\mathrm{C}$, Bueno $\mathrm{H}$, et al. 2017 ESC Guidelines for the management of acute myocardial infarction in patients presenting with ST-segment elevation: The Task Force for the management of acute myocardial infarction in patients presenting with ST-segment elevation of the European Society of Cardiology (ESC). Eur Heart J. 2018;39(2):119-77.

3. Szerlip M, Anwaruddin S, Aronow H, Cohen M, Daniels M, Dehghani P, et al. Considerations for cardiac catheterization laboratory procedures during the COVID-19 pandemic perspectives from the Society for Cardiovascular Angiography and Interventions Emerging Leader Mentorship (SCAI ELM) Members and Graduates. Catheter Cardiovasc Interv. 2020 Mar 25. doi: 10.1002/ccd.28887. [Epub ahead of print]

4. Jing ZC, Zhu HD, Yan XW, Chai WZ, Zhang S. Recommendations from the Peking Union Medical College Hospital for the management of acute myocardial infarction during the COVID-19 outbreak. Eur Heart J. 2020 Mar 31. doi: 10.1093/eurheartj/ehaa258. [Epub ahead of print] 